\title{
A Case Report of Mesenteric Panniculitis and Primary Sjögren's Syndrome
}

\author{
R. L. Batten, W. F. Ng \\ Musculoskeletal Research Group, Institute of Cellular Medicine, Newcastle University, Newcastle upon Tyne, Tyne and Wear, New- \\ castle, UK \\ Email: rebecca.batten@newcastle.ac.uk
}

Received September $12^{\text {th }}, 2013$; revised October $12^{\text {th }}, 2013$; accepted October $20^{\text {th }}, 2013$

Copyright (C) 2013 R. L. Batten, W. F. Ng. This is an open access article distributed under the Creative Commons Attribution License, which permits unrestricted use, distribution, and reproduction in any medium, provided the original work is properly cited.

\begin{abstract}
Mesenteric Panniculitis is a benign fibro-inflammatory process involving adipose tissue of the mesentery. It is characterised by fat necrosis, chronic inflammation and fibrosis, causing thickening and shortening of the mesentery. Patients may present with localised abdominal pain, abdominal mass, intestinal obstruction and ischaemic colitis. We report a case of mesenteric panniculitis causing abdominal pain in a patient with active Primary Sjögren's Syndrome. The rarity of this case makes it of interest. We review the current literature on mesenteric panniculitis and its association with connective tissue disease and inflammatory conditions. A 64-year-old Caucasian male presented in 1994 with dry mouth. A diagnosis of Primary Sjögren's Syndrome (PSS) was made on salivary gland biopsy. In 2010 he presented with an exacerbation of his eye symptoms, muscle pain and fatigue. He complained of abdominal pain and night sweating, but denied any weight loss or change in bowel habit. There was no significant past medical history other than PSS. On examination he had a small right submandibular node and mild synovitis at the right proximal interphalangeal joint and carpometacarpal joint. Examination of the abdomen showed marked umbilical tenderness but no organomegally. Blood tests at this time showed an active inflammation: CRP of $61(\mathrm{NR}<5 \mathrm{mg} / \mathrm{L})$, ESR $39(\mathrm{NR}<20 \mathrm{~mm} / \mathrm{s})$, strongly positive ENA Ro and La. IgG was elevated at 18.6 (NR 5.8 - 15.4), Complement was low at 0.17 (NR 0.18 - 0.6). An abdominal ultrasound scan demonstrated a $6 \times 3 \times 3 \mathrm{~cm}$ area of diffuse homogenous fat encasing some mesenteric vessels in the area of focal tenderness. CT abdomen and pelvis showed oedematous mesenteric fat and lymph nodes in the jejunal small bowel mesentery, consistent with mesenteric panniculitis. Laparoscopic biopsy was discussed with the surgical team, but was felt not indicated as risk outweighed potential benefit. The patient was treated with a 9-week reducing course of oral steroids. His abdominal symptoms resolved although CT abdomen showed little improvement in mesenteric panniculitis. A review of the literature suggests that currently there is no standard treatment and management should be guided by patient symptoms. Mesenteric Panniculitis is rare; as a result evidence for treatment is limited to individual case reports. There is no clear link between symptom improvement and radiological resolution of mesenteric panniculitis. It has, therefore been suggested that follow-up imaging should be limited to those with persistent symptoms. Overall the prognosis for mesenteric panniculitis is good, up to half of patients do not require treatment, and recurrence of symptoms is uncommon.
\end{abstract}

Keywords: Sjögren's Syndrome; Autoimmune Disease; Mesenteric Panniculitis

\section{Introduction}

Mesenteric Panniculitis is a benign fibro-inflammatory process involving adipose tissue of the mesentery [1]. It is characterised by fat necrosis, chronic inflammation and fibrosis, causing thickening and shortening of the mesentery [2]. The pathophysiology remains unclear, although it has been linked with malignancy - in particular lymphoma, gastrointestinal and genitourinary [3]. Patients with mesenteric panniculitis may present with localised abdominal pain, abdominal mass, intestinal obstruction and ischaemic colitis, however between $30 \%-50 \%$ remain asymptomatic [2].

We report a case of mesenteric panniculitis causing abdominal pain in a patient with active Primary Sjögren's Syndrome. The rarity of this case makes it of interest: We review the current literature on mesenteric panniculitis and its association with connective tissue disease and 
inflammatory conditions.

\section{Case Report}

\subsection{Background}

EM is a 64-year-old Caucasian male. His initial symptoms began in 1994, when he required multiple dental fillings and complained of dry mouth. A diagnosis of Primary Sjögren's Syndrome (PSS) was made on Salivary gland biopsy. His symptoms became more troublesome in 2003. He complained of dry eyes and mouth; wrist, back and neck pain; dry skin and profound fatigue. There were no significant symptoms of the respiratory, gastrointestinal, genitourinary or neurological systems at this time. His symptoms were well controlled with lubricating agents and NSAIDs as required.

\subsection{Progression of Disease}

In 2010, he presented with an exacerbation of his eye symptoms, increasing muscle pain and fatigue. He complained of abdominal pain and night sweating, but denied any weight loss or change in bowel habit. He also complained of increasing pain in his legs and lower back. He denied paraesthesia or loss of power. There was no significant past medical history other than PSS, in particular, no history of vascular disease. He holidayed in China in September 2009 but was well throughout his stay and on his return.

\subsection{Examination Findings}

On examination he had a small right submandibular node and mild synovitis at the right proximal interphalangeal joint and carpometacarpal joint. Examination of the abdomen showed marked umbilical tenderness but no organomegally. Straight leg raising was significantly reduced bilaterally, and there was altered sensation in the L5/S1 distribution bilaterally. He also had some tenderness at L5.

\subsection{Investigations}

Blood tests at this time showed an active inflammation with an elevated CRP of 61 (normal range $<5 \mathrm{mg} / \mathrm{L}$ ), ESR 39 (normal range $<20 \mathrm{~mm} / \mathrm{s}$ ), and strongly positive ENA Ro and La. IgG was elevated at 18.6 (normal range 5.8 - 15.4), Complement was low at 0.17 (normal range 0.18 - 0.6) Liver function tests and renal function were normal. Quantiferon gold test was positive, this was not pursued further. There was no evidence of malarial parasites on blood film. An abdominal ultrasound scan demonstrated a $6 \times 3 \times 3 \mathrm{~cm}$ area of diffuse homogenous fat encasing some mesenteric vessels in the area of focal tenderness. At this stage a CT was advised to further characterise this area.
CT abdomen and pelvis showed oedematous mesenteric fat and lymph nodes in the jejunal small bowel mesentery, consistent with mesenteric panniculitis.

CT PET confirmed a mild mesenteric panniculitis, but otherwise no significant abnormality.

The patient was reviewed by a neurologist who felt that his lower limb weakness and patchy sensory disturbance were most likely due to a radiculopathy, however a small fibre neuropathy could not be ruled out. Nerve conduction studies, CK and MRI lumbar spine were normal.

It was noted that IgA Tissue Transglutaminase 92.6U (normal range $0-15 \mathrm{U}$ ) and $\operatorname{IgA}$ endomysial antibody was positive. A GI Endoscopy was performed to rule out coeliac disease (associated with peripheral neuropathy). Duodenal biopsy was normal, with no increase in epithelial lymphocytes or abnormality of the villous architecture. Coeliac disease and TB were excluded.

Laparoscopic biopsy was discussed with the surgical team, but was felt not indicated as risk outweighed potential benefit.

\subsection{Management}

The patient was treated with a 9-week-reducing course of oral steroids. His abdominal symptoms resolved although CT abdomen showed little improvement in mesenteric panniculitis. The decision not to pursue further investigation was discussed and agreed by the patient as his symptoms improved and he had undergone a number of investigations already.

\section{Discussion}

Mesenteric Panniculitis is a non-specific inflammatory process, most commonly affecting the fatty tissue at the root of the small bowel mesentery, [4] resulting in thickening and shortening of the mesentery. The bowel, adjacent lymph nodes and vessels are usually not affected [2].

Little is known about the pathophysiology of mesenteric panniculitis. Characteristic histopathological findings include non-focal lymphoplasmocytic infiltration, fat cell necrosis, foamy macrophages and focal fibrosis. Schaffler et al. argue that adipocytes and adipocytokines are involved in primary inflammatory processes, and that mesenteric panniculitis is just one example of this [5].

There is a variety of terminology relating to the above process, which is based on the varied histological findings. These include mesenteric lipodystrophy (predominantly fatty necrosis), mesenteric panniculitis (Chronic inflammation), and retractile mesenteritis or mesenteric fibrosis (predominantly fibrosis). Emory et al. suggested the overarching terminology of sclerosing mesenteritis, under which the headings mesenteric panniculitis (predominant inflammation and fat necrosis) and retractile me- 
senteritis (predominant fibrosis and retraction) are recognised [6].

Such varied histology is likely to account for the varied clinical presentation. Documented presenting symptoms include abdominal pain, palpable mass, weight loss, small bowel obstruction, diarrhoea and chylous ascites [7, 8]. Occasionally it is asymptomatic, identified as an incidental finding on $\mathrm{CT}$ [3].

A recent systematic review (between 1980 and 2012) of 262 patients who were admitted with mesenteric panniculitis demonstrated that $69 \%$ were male, with a mean age of 63 years [8]. Similarly, Akram et al. reviewed 92 cases between 1982-2005, and found that 70\% were male, the mean age of onset was 65 years (55 - 72 years) [9]. The prevalence of mesenteric panniculitis was documented as $0.6 \%$ in one study: Daskalogianniki et al. prospectively reviewed over 7000 abdominal CTs, looking for features typical of mesenteric panniculitis. Of these, 49 demonstrated mesenteric panniculitis. 34 cases were associated with malignant disease, 11 cases associated with benign disease, and in the remaining 4 cases no association was identified [3].

Etiological associations of mesenteric panniculitis include malignancy, infectious disease, autoimmune disease, vasculitis, cirrhosis, peptic ulcer disease, pancreatitis, abdominal aortic aneurysm, previous abdominal surgery and elevated IgG4 related disease [3] (a fibroinflammatory condition initially associated with autoimmune pancreatitis, but now recognised in a number of conditions including atopic dermatitis, vasculitis, pancreatic cancer, biliary tract disease, and also present in $5 \%$ of the normal population) [10].

In terms of autoimmune disease, mesenteric panniculitis has been recognised in patients with autoimmune haemolytic anaemia [11], Coeliac disease, thyroiditis, primary sclerosing cholangitis, rheumatoid arthritis, lupus and polychondritis [12,13]. However to our knowledge, there is only one other case documenting an association with Sjogren's syndrome [14]. Like our case report, this patient presented with abdominal pain. She initially underwent surgery for acute appendicitis and ileocaecal abscess. She had a recurrence of her symptoms associated with annular rash and fever. Review of the histopathology suggested mesenteric panniculitis. She was treated with $20 \mathrm{mg}$ prednisolone daily and her symptoms resolved. CT also demonstrated complete resolution of the soft tissue density after 3 weeks of prednisolone treatment.

It is also worth noting that although our patient did not have clinical or histological evidence of coeliac disease, he did have raised IgA TTG and IgA endomysial antibody. There is a documented association between mesenteric panniculitis and coelic disease, supporting the link between mesenteric panniculitis and autoimmune disease.

Daskalogiannaki et al. found that of the 15 patients with non-malignant disease, only three had follow-up imaging. CT findings showed stable disease, that is, there was no progression to retractile mesenteritis or malignancy. Of the four biopsy-proven symptomatic patients, three were treated with prednisolone. Two showed a clinical improvement, and one had on-going intermittent abdominal pain. On follow-up CT, none of the patients had resolution or change in the mass [3]. The evidence for radiological improvement from other case reports following treatment is mixed $[9,13,15]$. This suggests that reimaging should be guided by patient symptoms in clinical practice.

There is no consensus on treatment of mesenteric panniculitis. Most would agree that treatment should be symptom-directed. Potential treatment options include surgical resection, immunomodulatory (steroids, azathioprine, cyclophosphamide), antifibrotic agents (thalidomide, [16] tamoxifen, [9] pentoxyfylline [17] NSAIDS (colchicine) [18] and conservative management. In this case report our patient showed a moderate response to prednisolone and no further intervention was required.

\section{REFERENCES}

[1] A. Daumas, S. Agostini, J. Villeret, P. Ah-Soune, O. Emungania and B. Granel, "Spontaneous Resolution of Severe, Symptomatic Mesocolic Panniculitis: A Case Report," BMC Gastroenterology, Vol. 6, No. 12, 2012, p. 59. http://dx.doi.org/10.1186/1471-230X-12-59

[2] G. Piessen, C. Mariette and J. P. Triboulet, "Mesenteric Panniculitis. [Article in French]," Annales De Chirurgie, Vol. 131, No. 2, 2006, pp. 85-90.

http://dx.doi.org/10.1016/j.anchir.2005.09.009

[3] M. Daskalogiannaki, A. Voloudaki, P. Prassopoulos, E. Magkanas, K. Stefanaki, E. Apostolaki, et al., "CT Evaluation of Mesenteric Panniculitis: Prevalence and Associated Diseases," AJR American Journal of Roentgenology, Vol. 174, No. 2, 2000, pp. 427-431.

http://dx.doi.org/10.2214/ajr.174.2.1740427

[4] G. Guettrot-Imbert, L. Boyer, J. C. Piette, I. Delèvaux, M. André and O. Aumaître, "Mesenteric Panniculitis. [Article in French]," La Revue de Médecine Interne, Vol. 33, No. 11, 2012, pp. 621-627. http://dx.doi.org/10.1016/j.revmed.2012.04.011

[5] A. Schäffler, U. Müller-Ladner, J. Schölmerich and C. Büchler, "Role of Adipose Tissue as an Inflammatory Organ in Human Diseases," Endocrine Reviews, Vol. 27, No. 5, 2006, pp. 449-467. http://dx.doi.org/10.1210/er.2005-0022

[6] T. S. Emory, J. M. Monihan, N. J. Carr and L. H. Sobin, "Sclerosing Mesenteritis, Mesenteric Panniculitis and Mesenteric Lipodystrophy: A Single Entity?" The American Journal of Surgical Pathology, Vol. 21, No. 4, 1997, pp. 392-398. http://dx.doi.org/10.1097/00000478-199704000-00004

[7] S. Khanna, S. Singh and D. Pardi, "Clinical Features and Outcomes in Hospitalized Patients with Sclerosing Mesenteritis," American Journal of Gastroenterology. Confe- 
rence: 77th Annual Scientific Meeting of the American College of Gastroenterology, Las Vegas, October 2012.

[8] T. Kerdsirichairat, A. Shaukat, H. Mesa and J. Abraham, "Natural History and Response to Treatments of Sclerosing Mesenteritis: Case Series and a Systematic Review," American Journal of Gastroenterology. Conference: 77th Annual Scientific Meeting of the American College of Gastroenterology, Las Vegas, October 2012.

[9] S. Akram, D. S. Pardi, J. A. Schaffner and T. C. Smyrk, "Sclerosing Mesenteritis: Clinical Features, Treatment, and Outcome in Ninety-Two Patients," Clinical Gastroenterology and Hepatology, Vol. 5, No. 5, 2007, pp. 589-596. http://dx.doi.org/10.1016/j.cgh.2007.02.032

[10] J. H. Ryu, R. Horie, H. Sekiguchi, T. Peikert and E. S. Yi, "Spectrum of Disorders Associated with Elevated Serum IgG4 Levels Encountered in Clinical Practice," International Journal of Rheumatology, Vol. 2012, 2012, Article ID: 232960. http://dx.doi.org/10.1155/2012/232960

[11] D. G. Robbrecht, F. Alidjan, B. Eikemans, D. A.Haans, C. van Guldener and P. van Wijngaarden, "Mesenteric Panniculitis: Variable Presentations [Dutch] Original Title Panniculitis Mesenterica: Uiteenlopende Presentaties," Nederlands Tijdschrift voor Geneeskunde, Vol. 155, No. 25, 2012, Article ID: A4555.

[12] S. Tyagi, M. Batke and L. Maas, "Celiac Sprue Presenting as Mesenteric Panniculitis," American Journal of Gastroenterology. Conference: 75th Annual Scientific Meeting of the American College of Gastroenterology, October 2010.

[13] K. Vlachos, F. Archontovasilis, E. Falidas, S. Mathioulakis, S. Konstandoudakis and C. Villias, "Sclerosing Mes- enteritis: Diverse Clinical Presentations and Dissimilar Treatment Options. A Case Series and Review of the Literature," International Archives of Medicine, Vol. 4, No. 2, 2011, p. 17. http://dx.doi.org/10.1186/1755-7682-4-17

[14] T. Sugihara, R. Koike, Y. Nosaka, J. Ogawa, H. Hagiyama, K. Nagasaka, et al., "Nihon Rinshō Men'eki Gakkai kaishi," Japanese Journal of Clinical Immunology, Vol. 25, No. 3, 2002, pp. 277-284. http://dx.doi.org/10.2177/jsci.25.277

[15] I. Issa and H. Baydoun, "Mesenteric Panniculitis: Various Presentations and Treatment Regimens," World Journal of Gastroenterology, Vol. 15, No. 30, 2009, pp. 38273830. http://dx.doi.org/10.3748/wjg.15.3827

[16] P. M. Ginsburg and E. D. Ehrenpreis, "A Pilot Study of Thalidomide for Patients with Symptomatic Mesenteric Panniculitis," Alimentary Pharmacology \& Therapeutics, Vol. 16, No. 12, 2002, pp. 2115-2122. http://dx.doi.org/10.1046/j.1365-2036.2002.01383.x

[17] A. N. Kapsoritakis, C. D. Rizos, S. Delikoukos, D. Kyriakou, G. K. Koukoulis and S. P. Potamianos, "Retractile Mesenteritis Presenting with Malabsorption Syndrome. Successful Treatment with Oral Pentoxifylline," Journal of Gastrointestinal and Liver Diseases, Vol. 17, No. 1, 2008, pp. 91-94.

[18] Fasoulas, K. Beltsis, A. Katsinelos, T. Dimou, et al., "Efficacy of Colchicine in the Treatment of Mesenteric Panniculitis in a Young Patient," Saudi Journal of Gastroenterology, Vol. 18, No. 2, 2012, pp. 146-148. http://dx.doi.org/10.4103/1319-3767.93825 